\title{
LA IDEA DE CIENCIA EN MIGUEL ANTONIO CARO
}

\author{
Leonardo Tovar \\ Universidad Santo Tomás
}

\section{Resumen}

El artículo analiza la concepción de Miguel Antonio Caro en torno a la ciencia. En el primer apartado se reseñan las críticas del pensador bogotano contra la filosofía positivista, que en sus tres versiones (el positivismo de Comte, el evolucionismo biológico de Darwin y el evolucionismo social de Spencer) pretende poner las hipótesis científicas por encima de las verdades de la religión. A continuación, se expone la doctrina católica del autor en torno al verdadero saber científico, que en coincidencia con Meléndez Pelayo, Caro estima necesariamente afín a la fe. Para concluir, se discute la visión reaccionaria de Caro sobre la ciencia moderna, ya que ésta ha estado en la base de la mentalidad anticientífica de la educación en Colombia.

\section{Palabras clave}

Miguel Antonio Caro, catolicismo, ciencia, positivismo, religión.

\section{Abstract}

The article analyses Miguel Antonio Caro's criterion concerning science. In the first section, Caro's critics against the positivist philosophy are pointed out; this one in its three versions: (Comte's positivism, Darwin's biological evolutionism and Spencer's social evolutionism) it seeks to set the scientific hypotheses above the truths of the religion. Next, the author's Catholic doctrine around the true scientific knowledge is exposed, which in coincidence with Meléndez Pelayo, Caro thinks similar to faith necessarily. To conclude, Caro's reactionary vision on the modern science is discussed, since this it has been on the basis of the anti-scientific mentality of the education in Colombia.

\section{Index terms}

Miguel Antonio Caro, catholicism, science, positivism, religion. 
En este artículo expondremos la concepción en torno a la ciencia sustentada por el humanista católico y político conservador Miguel Antonio Caro (1843- 1909), quien gobernó a Colombia entre 1892 y 1898. En el siglo XIX, don Miguel Antonio fue el más intransigente opositor de las ideas e instituciones de inspiración moderna impulsadas por los sectores liberales, y el más celoso defensor de la preeminencia de la fe y el clero católicos en la orientación del conocimiento y de la sociedad" ${ }^{1}$ En su criterio, "toda razón científica es buena, muy buena, subordinada al principio religioso, a la verdad; mala, muy mala, independiente, o subordinada al error $^{2}$ ".

\section{Críticas a las filosofías positivistas}

Para Caro, de ningún modo podía admitirse la pretensión positivista de absolutizar el saber científico y negar desde allí la validez de la religión y de la Iglesia. En su criterio, Auguste Comte, Charles Spencer y Charles Darwin eran exponentes de la detestada escuela experimental que él había combatido en sus tempranas manifestaciones sensualista y utilitarista.

Hasta donde hemos explorado, la primera referencia significativa al primero reposa en la introducción que en 1873 escribió a la antología de textos de su padre ${ }^{3}$, donde recuerda que “deslumbrado” por el progreso de los Estados Unidos e influido por "la lec- tura de algunas obras positivas y sansimonianas”, José Eusebio Caro se separó del cristianismo "arrimándose a la doctrina, o mejor escuela, de Augusto Comte”, pero al final, cita Miguel Antonio a José Eusebio en defensa de su honor religioso, declara que "es preciso volver al catolicismo" 4 .

Más adelante, nombra de nuevo a Comte y esta vez también a Spencer, en su reseña de la compilación de textos de Rafael Núñez que lleva por título “La Reforma Política”. Allí Caro, después de declarar moderadamente su desacuerdo con la afición del político cartagenero al estudio de la sociología, ya que ésta a menudo se compone de "teorías falsas”, termina por tachar con acritud el trabajo del sociólogo inglés: "De la [sociología] de Spencer (...), que es su más notable representante, podemos asegurar que consiste sólo en la explicación de los destinos de las sociedades basada en el examen de las tendencias del salvaje (a quién él erróneamente apellida hombre primitivo), mediante la recolección de datos muchas veces falsos, tomados de viajeros confrontados con no poca malicia para ridiculizar las ideas religiosas, y prescindiendo siempre, así del orden de la sociedad realmente primitiva, como de las tendencias del género humano rehabilitado por Jesucristo" "5. Y agrega Caro que una doctrina que desconoce tan flagrantemente el hecho esencial de la Revelación y la obra civilizadora de la Iglesia, mal puede aspirar al título de ciencia. Además, comenta

1 Sobre el pensamiento de M.A.C., ver en especial sus escritos compilados en: CARO, Miguel Antonio. Obras. Tomo I: Filosofía, religión, pedagogía. Edición de Carlos Valderrama Andrade. Bogotá: Instituto Caro y Cuervo, 1962.

2 CARO, Miguel Antonio. “Estudio sobre el utilitarismo”. 1869. En: Obras. Tomo I: Filosofía, religión, pedagogía. Bogotá: Instituto Caro y Cuervo, 1962, p. 154.

3 Véase: CARO, Miguel Antonio. "Introducción”. En: Obras escogidas en prosa y en verso, publicadas e inéditas, de José Eusebio Caro. Bogotá: Imprenta de El Tradicionista, 1873, pp. III- XLIV.

4 Ibíd., p. XXXVIII.

5 CARO, Miguel Antonio. “La reforma política”. 1885. En: Escritos políticos. Segunda serie. Bogotá: Instituto Caro y Cuervo, 1990, p. 84. 
burlonamente que "Spencer sólo se acuerda de Colombia” 6 para informar que aquí hay gentes que creen descender de cierto tipo de ratones.

No obstante estos comentarios negativos, enseguida Caro disculpa a Núñez por leer “con gusto a Spencer”, pues “¿quién no lee con gusto a un fantaseador ingenioso?”, pero aclara para que no queden dudas que los métodos sociológicos de uno y otro son diametralmente diferentes: "Spencer es naturalista, considera a la sociedad animadamente, y funda la sociología en la biología; Nüñez es espiritualista, tiene profunda fe en el gobierno temporal de la Providencia"8. En consecuencia, con beneficio para la ortodoxia de su socio político, deduce Caro que cuando Núñez habla de sociología lo hace en el sentido noble de "filosofía social fundada en el estudio de la historia”, acepción en la cual “José de Maistre sería el primer sociólogo del siglo XIX y Herbert Spencer (...) sería el mayor enemigo de la sociología”`. Sin embargo, admite el reseñador que el término sociología ya posee un significado convencional generalmente aceptado que "nació con odioso sello de los labios materialistas de Augusto Comte y ha sido monopolizado por la escuela positivista” ${ }^{10}$.

En tercer lugar, recordemos la extensa nota que le dedica a Spencer en la introducción a su antología de "Artículos y discursos"1, donde recrimina la ignorancia de los filósofos contemporáneos no católicos en cuestiones teológicas. Por ejemplo, el pensador in- glés acertadamente se opone a Hobbes y a Bentham al reconocer la existencia de un derecho natural que sirve de dique a la omnipotencia parlamentaria, pero basado en una cita incompleta de Bossuet, se equivoca al incluir a los teólogos católicos entre los defensores del poder absoluto de la autoridad temporal. "Si Spencer acertase a hojear a Santo Tomás, a Soto, a cualquiera de los grandes teólogos católicos (...) en Bossuet mismo (...) hallaría sabiamente limitado el poder civil, y establecida la diferencia entre el legítimo imperante y el tirano” ${ }^{12}$. En un inglés, esta desinformación es tanto más atrevida, por cuanto el rompimiento entre Enrique VIII y la Santa Sede se produjo precisamente porque ésta no consintió con el poder absoluto "sin límite moral alguno" al que aspiraba aquel. La sorpresa que nos cause por qué un político autoritario como Caro aplaude las limitaciones al poder se atenúa cuando reparamos en que sus dardos van dirigidos en primer lugar contra la "omnipotencia parlamentaria”, y cuando añade líneas después que si bien la ley natural y revelada restringe la autoridad temporal, “en pueblos cristianos no hay necesidad de ocurrir al derecho natural, porque la Iglesia lo interpreta y complementa en sus enseñanzas”13.

La reiteración que hace al final de esta nota Caro a su repudio al método de Spencer de valerse del testimonio de los salvajes para explicar la sociedad cristiana de Europa, nos sirve de puente para glosar sus anotaciones sobre Darwin, pues, para nuestro autor, menos aceptable aún era hablar del hombre

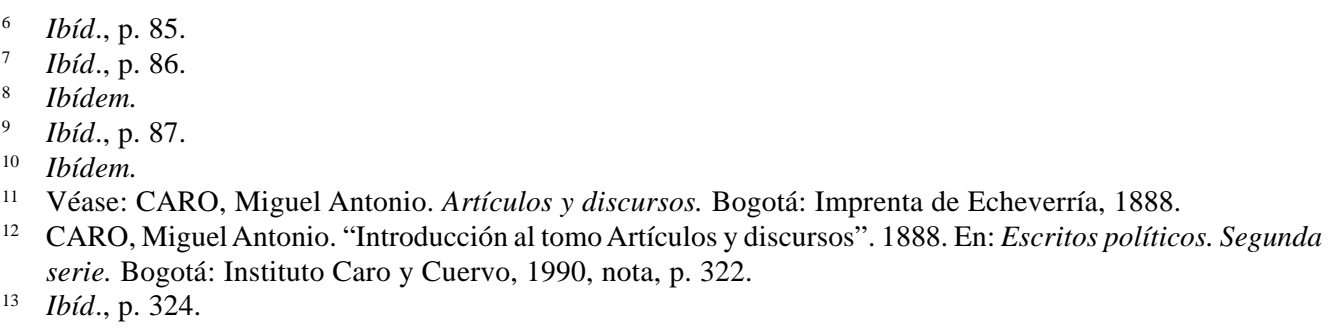


equiparándolo a los animales. En una serie de artículos de 1882 y 1883 dedicada a refutar a un articulista liberal que se vanagloriaba del supuesto atraso científico de España ${ }^{14}$, el intelectual católico exclama que "siendo nosotros raza española”, este defecto iría en detrimento de la cultura nacional: “ ¿ $[\mathrm{N}] \mathrm{o}$ andan por ahí también quienes desearían que saliese cierta la teoría darwiniana para tener la satisfacción de descender indisputablemente del mono? ¡Vaya que hay gustos depravados!”15.

En la reseña panfletaria ${ }^{16}$ que escribió años después para fulminar las tesis evolucionistas de su otrora amigo Jorge Isaacs ${ }^{17}$, Caro extrema sus prevenciones contra esa ciencia “antirreligiosa y antihumana”. Basado en la refutación irrefutable que opuso De Maistre “como jugando” a los razonadores del siglo XVIII ("los más enclenques razonadores de todos los siglos”) que anticiparon a Darwin, Caro falla que la teoría darwiniana "no es un hecho histórico”, pues el paso del mono al hombre no se ha verificado de ningún modo $^{18}$.

\section{La concepción de ciencia}

En los artículos sobre la ciencia española de 1882 y 1883, el ideólogo conservador declara que en tanto conjunto de conocimientos e inventos útiles, la ciencia es "un acervo común” de la humanidad que no le pertenece en exclusividad a ninguna nación y que avanza continuamente por aportes provenientes de todas las latitudes ${ }^{19}$. De allí que no sólo las españolas, como quieren ligeramente los críticos liberales, sino todas las obras científicas del pasado pueden quemarse sin que ello haga mella en la conservación del saber alcanzado por el hombre: “...para eso cualquier enciclopedia vale más que la Sagrada Escritura" ${ }^{20}$. Al fin de cuentas, los libros son apenas el testimonio literario del avance científico, de modo que la pérdida de los textos españoles no reduciría la ciencia a cenizas, pero en contrapartida ellos permitirían salvarla en caso de que sucediera "un naufragio general de las demás naciones”21.

Por otra parte, como lo ha probado Meléndez Pelayo de manera irrefutable, no se puede

14 Publicados originalmente en El Conservador de Bogotá, en respuesta al Diario de Cundinamarca y otros órganos liberales, la serie está integrada por los artículos "El atraso español”, "La ciencia española”, "La ciencia española y la Inquisición” y "Procedimientos de la inquisición española”.

15 CARO, Miguel Antonio. "El atraso español”. 1882. En: Ideario hispánico. Bogotá: Instituto Colombiano de Cultura Hispánica, 1952, p. 147.

16 CARO, Miguel Antonio. “El darwinismo y las misiones”. Repertorio Colombiano (Bogotá) No.VI, febrero de 1887, pp. 464- 491; continuado en No. VII, marzo de 1887, pp. 5-35; recogido por Carlos Valderrama Andrade en CARO, Miguel Antonio. Obras. Tomo I: Filosofía, religión, pedagogía. Bogotá: Instituto Caro y Cuervo, 1962, pp. 1049-1107.

17 Véase: ISAACS, Jorge. “Estudio sobre las tribus indígenas del Magdalena, antes provincia de Santa Marta”. Anales de Instrucción Pública (Bogotá), septiembre de 1884, vol. 45, pp. 178-352. Edición contemporánea en Bogotá: Incunables, 1983.

18 Para un estudio más amplio del juicio de Caro sobre Darwin, puede consultarse nuestra contribución al libro Reexamen de Caro, próximo a salir.

19 CARO, Miguel Antonio. “La ciencia española”. 1882. En: Ideario hispánico. Bogotá: Instituto Colombiano de Cultura Hispánica, 1952, p. 154.

20 Ibíd., p.155.

21 Ibíd., p. 156. 
desconocer el papel fundamental que jugó el cristianismo en el desarrollo de la ciencia europea tomada como una unidad que ni siquiera la herejía protestante logró disolver, ni menos ignorar el liderazgo español en este proceso con figuras como Francisco Suárez. En la nota publicada por la misma época contra el prefacio que escribió Murillo Toro a la edición bogotana de la obra del historiador norteamericano J. W. Draper sobre los "supuestos" conflictos entre la religión y la ciencia $^{22}$, Caro afirma categóricamente que "La ciencia es, como el entendimiento humano, naturalmente religiosa" ${ }^{23}$, e invocando a su padre añade que si bien las verdades científicas no son dogmas religiosos, "el cristianismo es indirectamente, por su influencia fecunda, autor y propagador de las ciencias”24.

Y si de reclamar actualización científica se trata, los que menos ejemplo pueden dar son los liberales colombianos, quienes aún recomiendan los textos de Bentham y Tracy, traducidos al español a comienzos del siglo XIX. "Solo en Colombia, en virtud de ciertas ideas 'progresistas', hay textos que viven y perduran luengos años aunque en todas partes ya han envejecido y míseramente caducado por el curso natural de las cosas" ${ }^{25}$. Si esos comentaristas califican a Meléndez Pelayo como ultramontano y medieval, anota sarcásticamente don Miguel Antonio, no tenemos más remedio que considerarlos a ellos mismos como representantes del pensamiento antiguo.
Frente a la acusación de los librepensadores peninsulares repetida aquí por sus copartidarios colombianos en torno a que la intolerancia clerical tuvo la culpa de que España no hubiera engendrado creadores de ciencia comparables a Bacon, Descartes, Newton, Leibniz, Euler, Laplace, Torricelli, Humboldt, Volta, Daguere, Morse, Buffon, Lamarck, etc., nuestro autor reta a sus opositores a que le enumeren las cumbres científicas que han surgido de épocas heterodoxas. En realidad, estas figuras cimeras de la ciencia sólo surgen de cuando en cuando, sin que en ello hayan repercutido la tolerancia o la intolerancia. “(...) si no tuvimos Galileos, Newtones o Kepleros, será por otra razón, porque la Inquisición no los prohi$b i o^{26}$. Más que dolerse porque España no fue la patria de los genios oriundos de otros países, se trata de comprender que si bien todas las naciones poseen en común el patrimonio científico de la humanidad, cada una ha sobresalido en un campo particular, y por tanto nada tienen que envidiarse una a otra. A quienes echan de menos los descartes, newtones o bacones hispanos, podría inquirírseles por qué Francia, Alemania e Inglaterra unidas no han podido producir "ni un solo Meléndez Pelayo"27.

En su ímpetu ortodoxo, Caro ha perdido el sentido de las proporciones por completo, $\mathrm{y}$ lleva su dialéctica polemista al extremo de desconocer el valor de los hitos de la ciencia y la filosofía modernas. El método inductivo es un invento de Aristóteles, “a quien Bacon

\footnotetext{
22 Véase: DRAPER, J. W. “Los conflictos entre la ciencia y la religión”. Bogotá: Edición de José Benito Gaitán, 1878. Biblioteca de El Diario de Cundinamarca. El prefacio de Manuel Murillo Toro, sin firma, pP. IIII - XII.

23 CARO, Miguel Antonio. “Recuerdos y rectificaciones”. 1882. Obras. Tomo I: Filosofía, religión, pedagogía. Bogotá: Instituto Caro y Cuervo, 1962, p. 964.

${ }^{24}$ Ibíd., p. 970.

25 CARO, Miguel Antonio. “La ciencia española”. 1882. En: Ideario hispánico. Bogotá: Instituto Colombiano de Cultura Hispánica, 1952, pp. 153 y 154.

26 Ibíd., p. 164.

27 Ibíd., p. 165.
} 
refutó sin entenderlo bien”28. Si lo hubiera formulado un autor español, el entimema [sic] cartesiano sería considerado una perogrullada, pero "no es ciencia, ni vieja ni nueva”29. Además, ni el método de uno ni la duda del otro han originado "los modernos inventos mecánicos que tanto nos fascinan”30. Y en fin, "la teoría transformista predarwiniana de Lamarck no fue ni será ciencia sino una hipótesis peregrina buena para tejer sobre ella unas nuevas Metamorfosis ovidianas”31.

\section{La preeminencia de la fe}

Para lanzar sus anatemas, al integrista Caro le bastaba con la evidencia palpable del anticatolicismo de científicos y filósofos. En uno de los artículos en defensa de la ciencia española, después de aludir a los numerosos autores españoles que han contribuido al conocimiento y a la civilización, el gramático bogotano advierte que "en lo que es pobrísima la literatura española es en obras heterodoxas. Los de nuestra raza no sirven para herejes y hacen tristísimo papel como librepensadores”32. Con evidente agudeza polémica, su contradictor liberal de El diario de Cundinamarca parafrasea este pasaje diciendo que el escritor de El conservador ha reconocido que "el pensamiento español está como enfermo en el terreno de la indagación libre”. Sin embargo, Caro no se deja amilanar por esta vuelta de tuerca de su crí- tica antimoderna, y en la contrarréplica arguye que él no ha dicho ni pensado tal idea. "Para nosotros, la herejía no es indagación libre, sino ignominiosa servidumbre”33.

Paradójicamente, estas expresiones se lanzan a nombre de "los intereses de la ciencia, imparcial y severa” ${ }^{4}$. Para él "la religión verdadera y la ciencia verdadera no pueden hallarse en contradicción”35, pues cada una posee su propia esfera que no debe ser invadida por la otra. Ni las verdades científicas deben buscarse en la Biblia, ni las verdades morales y religiosas se hallarán en los textos científicos. Pero a pesar de ese aparente trato equivalente, la prelación de la fe es clara: “(...) lo que no puede admitirse teológicamente es que una tesis puramente científica, aunque esté demostrada, cuanto más si es dudosa, como lo era la del sistema copernicano, se sostenga y profese a título de verdad teológica”36.

Si casi tres siglos después justifica la condena de la Iglesia al heliocentrismo de Galileo, con mayor motivo desprecia Caro el evolucionismo darwinista, que de acuerdo con Pasteur y otros naturalistas carece de cualquier respaldo en los hechos. En contravía de esa hipótesis que niega la dignidad del hombre y la bondad de la Revelación, la prueba concluyente de la armonía entre ciencia y creencia religiosa reside para él en que los sabios más valiosos del mundo son católi-

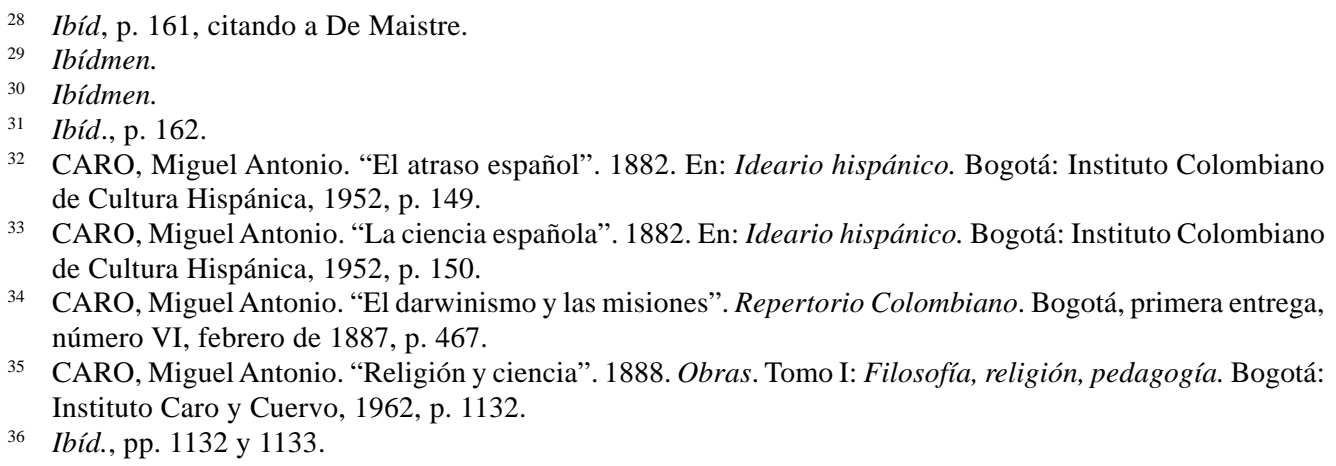

33 CARO, Miguel Antonio. “La ciencia española”. 1882. En: Ideario hispánico. Bogotá: Instituto Colombiano de Cultura Hispánica, 1952, p. 150.

34 CARO, Miguel Antonio. “El darwinismo y las misiones”. Repertorio Colombiano. Bogotá, primera entrega, número VI, febrero de 1887, p. 467.

35 CARO, Miguel Antonio. "Religión y ciencia”. 1888. Obras. Tomo I: Filosofía, religión, pedagogía. Bogotá: Instituto Caro y Cuervo, 1962, p. 1132.

36 Ibíd., pp. 1132 y 1133. 
cos, y como ejemplo de ello nombra al médico positivista Claude Bernard.

Nótese que Caro tergiversa el sentido de la compatibilidad entre ciencia y fe. Por mencionar sólo dos referentes de épocas y lugares distantes, en Alberto de Colonia y en José Celestino Mutis, la indagación científica poseía dignidad intrínseca como medio de ejercer la razón natural del hombre y acercarlo a la obra del Creador, y por tanto debía respetarse su independencia metodológica. En cambio, el reaccionario Caro reclamaba la completa sujeción de la ciencia a los dogmas eclesiásticos. Con dicha mentalidad enquistada en las instituciones ${ }^{37} \mathrm{y}$ en la educación, no es de extrañar que hayamos carecido de desarrollo científico, pero al cabo quizás el mayor perjuicio lo haya sufrido una religión ostentosamente anticientífica.

37 No se olvide que M.A.C. fue el redactor de la Constitución Política que rigió a Colombia entre 1886 y 1991. 